\title{
Enhancement of Power System Flexibility and Operating Cost Reduction Using a BESS
}

\author{
Ioannis Papayiannis, Markos Asprou, Lysandros Tziovani, and Elias Kyriakides \\ Department of Electrical and Computer Engineering, KIOS Research and Innovation Center of Excellence \\ University of Cyprus \\ Nicosia, Cyprus \\ \{papayiannis.ioannis, asprou.markos, tziovani.lysandros, elias\}@ucy.ac.cy
}

\begin{abstract}
Power system operators are continuously striving to achieve more economical, environmental friendly, and stable operation of the system. The integration of Renewable Energy Sources (RES) can benefit the system in terms of cost and environmental friendliness but at the same time it poses challenges related to system stability. One way for achieving an increased penetration of RES while maintaining the system integrity is the installation of Battery Energy Storage Systems (BESS). In this paper, the effect of a large scale BESS on the power system operating condition is investigated. A MILP-based unit commitment algorithm is used for obtaining the optimal generation scheduling in the system when a BESS is employed and the results are used for evaluating the improvement in several indices such as system operating cost, $\mathrm{CO}_{2}$ emissions, and system flexibility. Through a case study, the positive impact of the deployment of a BESS on a power system is verified, indicating that the aforementioned indices are improved in the presence of a BESS.
\end{abstract}

Index Terms - Battery energy storage system, insufficient ramping resource expectation, renewable energy sources, system flexibility.

\section{I.INTRODUCTION}

The rapid climate change that is even more apparent in the last decades, motivate the scientific community to promote environmental friendlier technologies and practices. One of the reasons that inevitably accelerated the environmental pollution is the imprudent emit of the so-called Greenhouse Gases (GHG), such as $\mathrm{CO}_{2}$ and $\mathrm{NO}_{\mathrm{x}}$, which are produced by the combustion of fossil fuels, mainly in the electricity and transport sectors. Regarding the electricity sector, new sustainable technologies involving the use of renewable energy sources (RES), such as solar and wind power, are highly integrated into modern power systems.

The variable generation of RES, due to the inherently unpredictable and intermittent nature of wind and solar, causes several challenges to the power system operation (especially when the RES penetration is high), for maintaining the supply-demand equilibrium. Actually, the capability of the system to maintain the balance between the load and the demand even under high RES power fluctuation, is called flexibility. Flexibility in power systems can be provided by thermal generating units, energy storage systems (ESSs), interconnections, demand response, etc [1], [2]. In some extreme cases, such as isolated systems without any hydropower plants, flexibility is only available from thermal units.

This paper is part of the FLEXITRANSTORE project. This project has received funding from the European Union's Horizon 2020 research and innovation programme under grant agreement No 774407). This work has been also supported in part by the European Union's Horizon 2020 research and innovation programme under grant agreement No 739551 (KIOS CoE)
In such cases, the increasing capacity of RES and the fact that most of the flexibility resources are scheduled to be installed in a long-term planning scheme might lead to a system flexibility shortage. In this sense, an energy storage system (ESS) could be a viable solution for the provision of flexibility since it is easily integrated into a power system and it is a quite flexible power source [3], [4]. Furthermore, the deployment of BESS could reduce GHG emissions and the system's operating cost since it increases the RES penetration (by avoiding the need for curtailments), while it leads to more efficient operation of the thermal units.

In several works, the importance of flexibility in modern power systems with an increasing RES penetration level has been underlined, discussing the economic, security and power quality issues related to flexibility [2], [5], [6]. However, there is no unique definition for flexibility evaluation; in [7], a composite flexibility metric (CFM) is presented and used in unit commitment (UC) as a constraint to provide the desired flexibility at each interval, while in [8] a risk-based method is proposed to evaluate the flexibility by calculating the Insufficient Ramping Resource Expectation (IRRE).

Additionally, several studies have been conducted to evaluate different flexibility resources. The provision of flexibility through demand response management is examined in [9], where downward flexibility is provided by converting wind power to gas. In [10], the flexibility is provided by demand response when economic incentives are applied. Furthermore, the flexibility provided through the ESSs receives increasing attention lately. The minimum required flexibility is guaranteed by using the CFM as a UC constraint in [3], where a BESS is deployed for 48 hours in the power system of Cyprus. In [6], compressed air energy storage is evaluated as a flexibility resource over a 24 -hour horizon on a test system.

Greater flexibility enables the system to operate at a more efficient state, while the capability of absorbing green energy is significantly improved. In [1], an economic dispatch (ED) method, taking into account emission tax and utilizing BESS is proposed and evaluated on a simplified power system. The UC problem constrained by the CFM is solved for two days and the economic benefit is evaluated in [3]. The RES penetration and operating cost are considered in [4], where a BESS is employed in a small island's power system.

In this paper, an optimal operating strategy for a power system with high RES capacity and a BESS is considered. A 
UC and ED model [3] is employed for planning the optimal set of generators, aiming at reducing the operating cost, while several BESS capacities are considered to evaluate their impact on the cost and $\mathrm{CO}_{2}$ emissions. Further, the system capability to accommodate RES, when a BESS is employed, is evaluated under several RES penetration scenarios. The IRRE is also calculated to evaluate the system's flexibility per scenario, using a modified resources model to include BESS. One of the contributions of this work is the performance of the case studies using real data from an islanded power system, therefore the results presented in this paper reflect realistic conditions. Further, most of the case studies in the literature focus on the cost savings using the BESS while in this work the possible reduction in $\mathrm{CO}_{2}$ is investigated. Moreover, the impact of a BESS on the flexibility of a real power system is evaluated using a more representative method.

This paper is organized as follows. Section II gives a brief insight into the UC mathematical formulation. In Section III, the IRRE calculation methodology, considering $\mathrm{BESS}$, is presented. A case study and the operating cost, $\mathrm{CO}_{2}$ emissions and flexibility improvements are presented in Section IV. Finally, the main conclusions of this work are outlined in Section V.

\section{UC-EC MATHEMATICAL FORMULATION}

In this work, the unit commitment problem is formulated as a mixed-integer linear program (MILP) along an arbitrary time horizon $T$, with one-hour time intervals [3]. The quadratic cost function of the generating units is approximated by a piece-wise linear function in order to use the MILP technique to solve the problem. Finally, the UC solution is used as input to calculate the system's $\mathrm{CO}_{2}$ emissions.

\section{A. Objective Function}

The objective function that must be minimized (by choosing the optimal set of generators) to achieve minimum operating cost, is:

$$
\min f(\text { cost })=\sum_{\forall t \in T} \sum_{\forall i \in U}\left(F_{i}\left(p_{i}^{t}\right)+b_{i}^{t} N_{i}+z_{i}^{t} S_{i}\right)
$$

where, $T$ and $U$ represent the time periods (hours) and the number of generating units, respectively; $p_{i}^{t}$ is the power output, $b_{i}^{t}$ is the binary state, and $z_{i}^{t}$ is the start-up state of unit $i$ at observation $t . F_{i}\left(p_{i}^{t}\right)$ is the piece-wise linear cost function of unit $i$ at observation $t$, when power output is $p_{i}^{t}$; $N_{i}$ and $S_{i}$ are the no-load cost and the start-up cost of unit $i$, respectively.

In particular, the generation and start-up cost of the generating units is minimized for the whole considered period. In this work, zero marginal cost is assumed for RES and a strict priority dispatch is maintained. In addition, RES curtailments are performed when needed to ensure power system stability.

\section{B. Constraints}

The objective function subjects to the following constraints.

1) Generation Limits \& Units Start-up: The generated power from each committed unit must be within a range de- fined by their minimum $\left(P_{i}^{\text {min }}\right)$ and maximum $\left(P_{i}^{\text {max }}\right)$ operating limits. The generated power from the decommitted units is zero. In this sense, $p_{i}^{t}$ is a discontinued variable expressed in (2). The start-up state $\left(z_{i}^{t}\right)$ is set to 1 only when the corresponding unit is OFF at $t-1$ and $\mathrm{ON}$ at $t$ based on (3). In this manner, the start-up cost is included in the optimization problem.

$$
\begin{aligned}
p_{i}^{t} \leq P_{i}^{\max } \times b_{i}^{t}, & \forall i, \forall t \\
p_{i}^{t} \geq P_{i}^{\min } \times b_{i}^{t}, & \forall i, \forall t \\
-b_{i}^{t-1}+b_{i}^{t} \leq z_{i}^{t}, & \forall i, \forall t \in[2, T]
\end{aligned}
$$

2) Power Balance: The produced power from the committed units plus the RES generation $\left(p_{\text {Res }}^{t}\right)$ and the discharging power $\left(p_{D i s}^{t}\right)$ of the BESS must be equal to the load demand $\left(D^{t}\right)$ plus the charging power $\left(p_{C h}^{t}\right)$ of the BESS at each observation $t$. $p_{\text {Res }}^{t}$ is the usable RES generation, which is equal to the estimated generation $\left(\bar{p}_{\text {Res }}^{t}\right)$ when no operating constraints are violated.

$$
\begin{array}{cc}
\sum_{\forall i \in U} p_{i}^{t}+p_{\text {Dis }}^{t}+p_{\text {Res }}^{t}=p_{C h}^{t}+D^{t}, & \forall t \\
0 \leq p_{\text {Res }}^{t} \leq \bar{p}_{\text {Res }}^{t}, & \forall t
\end{array}
$$

3) Spinning Reserve: The available power from the committed units plus the RES power must be greater than the $D^{t}$ plus the spinning reserve $\left(R^{t}\right)$, as shown in (5). In this work, the battery storage does not contribute in the supply of reserve.

$$
\sum_{\forall i \in U} b_{i}^{t} \times P_{i}^{\max }+p_{R e s}^{t} \geq D^{t}+R^{t}, \quad \forall t
$$

4) Minimum $U p \&$ Down time: The minimum time a unit must stay online is the minimum uptime $\left(U p_{i}\right)$. On the other hand, when a unit is decommitted it must stay offline for a period defined by its minimum downtime $\left(D p_{i}\right)$ specification.

$$
\begin{gathered}
b_{i}^{t}-b_{i}^{t-1} \leq b_{i}^{T_{u p}}, \\
\forall i, T_{\text {up }} \in\left[t+1, \min \left\{t+U p_{i}-1, T\right\}\right], \\
\forall t \in[2, T-1] \\
b_{i}^{t-1}-b_{i}^{t} \leq 1-b_{i}^{T_{\text {down }}}, \\
\forall i, T_{\text {down }} \in\left[t+1, \min \left\{t+D p_{i}-1, T\right\}\right], \\
\forall t \in[2, T-1]
\end{gathered}
$$

5) State of Charge: The state of charge $\left(S O C_{t}\right)$ of the BESS at time $t$ is measured in MWh and is expressed as the initial capacity $(I C)$ of the storage minus the summation of the discharging power plus the summation of the charging power for all the past and the present time intervals. The $S O C_{t}$ must be within the minimum $\left(S O C_{\text {min }}\right)$ and maximum $\left(S O C_{\max }\right)$ state of charge. The SOC constraint is described in (8). The $s_{d}$ and $s_{c}$ are the discharging and charging coefficients of the battery, respectively.

$$
S O C_{t}=I C-1 / s_{d} \sum_{\tau=1}^{t} p_{D i s}^{\tau}+S_{C} \sum_{\tau=1}^{t} p_{C h}^{\tau}, \quad \forall t
$$




$$
S O C_{t} \geq S O C_{\text {min }}, \quad S O C_{t} \leq S O C_{\text {max }}, \quad \forall t
$$

6) Battery Storage Restriction: The BESS cannot charge or discharge within the same time interval and this is achieved through the constraints (9) and (10). Also, the charging and discharging power in MW must be less than the maximum charging $\left(P_{C h}^{\max }\right)$ and discharging $\left(P_{D i s}^{\max }\right)$ capability of the BESS (9). Note that $v_{t}$ and $n_{t}$ are binary variables associated with the discharging and charging power, respectively.

$$
\begin{array}{r}
0 \leq p_{D i s}^{t} \leq P_{D i s}^{\max } \times v_{t}, \quad \forall t \\
0 \leq p_{C h}^{t} \leq P_{C h}^{\max } \times n_{t}, \quad \forall t \\
v_{t}+n_{t} \leq 1, \quad \forall t
\end{array}
$$

It should be noted that the ramp-up and ramp-down constraints of the generating units are ignored in this study, since the units can change their production from the minimum to the maximum and vice versa, in the considered time intervals.

\section{Fuel Consumption and $\mathrm{CO}_{2}$ Emissions}

Once the UC is executed for the considered period, the operating cost is calculated along with the resulting $\mathrm{CO}_{2}$ emissions. The calculation of $\mathrm{CO}_{2}$ emissions is directly related to the fuel consumption, which for unit $i$ is given by (11), while the total $\mathrm{CO}_{2}$ emissions $(G)$ are given by (12).

$$
\begin{gathered}
L_{i}\left(p_{i}^{t}\right)=a_{i}+b_{i} p_{i}^{t}+c_{i}\left(p_{i}^{t}\right)^{2} \\
G=\sum_{\forall t \in T}\left(\gamma \times \sum_{\substack{\forall i \in U_{H} \\
U_{H}, U_{G} \subset U}} L_{i}\left(p_{i}^{t}\right)+\delta \times \sum_{\forall j \in U_{G}} L_{j}\left(p_{j}^{t}\right)\right),
\end{gathered}
$$

where, $a_{i}, b_{i}$, and $c_{i}$ are the fuel coefficients of each unit. $U_{H}$ is the subset of units using heavy fuel oil and $U_{G}$ are the units using gas oil; $\gamma$, and $\delta$ are the emitted tons of $\mathrm{CO}_{2}$ per fuel ton for each fuel type.

\section{IRRE CALCULATION}

The flexibility analysis in this paper involves the calculation of the IRRE, which expresses the expected number of observations of flexibility shortage in a considered time horizon [8]. In its original form, IRRE considers only flexibility provided by dispatchable, open cycle generating units (i.e., thermal and hydroelectric units). However, BESS is an available flexibility resource and the methodology for IRRE calculation is modified to take BESS into account. This is one of the innovation of this work.. The calculation of the IRRE involves 2 main steps:

1) Data Preprocessing: The net load, $N D$, is used for calculating the positive, $N D R_{h,+}^{t}$, and negative, $N D R_{h,-}^{t}$, ramps per time horizon $\mathrm{h}$ as shown in (13)-(15).

$$
\begin{gathered}
N D R_{h}^{t}=N D^{t}-N D^{t-h}, \quad 1 \leq t \leq \overline{\overline{N D}}-h \\
N D R_{h,+}^{t}=N D R_{h}^{t}, \quad \forall N D R_{h}^{t}>0 \\
N D R_{h,-}^{t}=N D R_{h}^{t}, \quad \forall N D R_{h}^{t}<0
\end{gathered}
$$

where, $\overline{\bar{N}} \overline{\bar{D}}$ is the number of observations in the dataset.
2) Resource Flexibility: The flexibility that a unit can provide is limited by its operational limits. A unit that operates at $P^{\max }$ or is out for maintenance cannot provide any upward flexibility. Additionally, no upward flexibility is available when the unit is decommitted and the start-up time is greater than the time horizon considered for flexibility calculation. In this sense, one unit can provide upward flexibility when the time horizon is greater than its start-up time or it operates below $P^{\max }$. Equation (16) describes the flexibility provided by thermal units, subject to (17)-(18).

$$
\begin{aligned}
& U F_{i, h}^{t}=R U_{i} \times\left(h-\left(1-b_{i}^{t}\right) \times S t_{i}\right. \\
\text { s.t. } & p_{i}^{t}+U F_{i, h}^{t} \leq P_{i}^{\text {max }} \\
& p_{i}^{t}+U F_{i, h}^{t} \notin\left(0, P_{i}^{\text {min }}\right)
\end{aligned}
$$

where, $R U_{i}$ is the ramp-up rate and $S t_{i}$ is the start-up time of unit $i$. Similarly, the downward flexibility on outage or when the unit operates at $P^{\mathrm{min}}$ is zero. In any other case, the downward flexibility is calculated by (19), subject to (20)(21), where $R D_{i}$ denotes the ramp-down rate of unit $i$.

$$
\begin{gathered}
D F_{i, h}^{t}=R D_{i} \times h \times b_{i}^{t} \\
\text { s.t. } p_{i}^{t}-D F_{i, h}^{t} \geq 0 \\
p_{i}^{t}-D F_{i, h}^{t} \notin\left(0, P_{i}^{\text {min }}\right)
\end{gathered}
$$

In order to take into account the flexibility available by a BESS in the IRRE calculation methodology, its SOC and operating state are required. The BESS's power output, depending on the technology, can be quite stable when the SOC is high enough. In this work, it is assumed that the BESS can operate at full output when the SOC is above $20 \%$. If less energy is available, then zero upward flexibility is available from the BESS. On the other hand, the downward flexibility is provided by charging the BESS at maximum power when the SOC is less than $80 \%$. The charging power is approximated as a linear function of the SOC when it is higher than $80 \%$. The flexibility of the battery in both directions is given by (22)-(23),

$$
\begin{gathered}
B U F^{t}\left(S O C^{t}\right)=\left\{\begin{aligned}
0, & S O C^{t}<0.2 \\
p_{C h}^{t}-p_{D i s}^{t}+P_{D i s}^{\max }, &
\end{aligned}\right. \\
B D C^{t} \geq 0.2
\end{gathered}
$$

The total upward and downward available flexibility provided by both the dispatchable generating units and battery is given by (24)-(25).

$$
\begin{aligned}
U F_{\text {system }, h}^{t} & =\sum_{\forall i \in U} U F_{i, h}^{t}+B U F^{t}\left(\operatorname{SOC}^{t+h}\right) \\
D F_{\text {system }, h}^{t} & =\sum_{\forall i \in U} D F_{i, h}^{t}+B D F^{t}\left(S O C^{t+h}\right)
\end{aligned}
$$

After calculating the flexibility provided by the resources of the system, the available flexibility distribution (AFD) is calculated from the system's flexibility time series using the Kaplan-Meier estimator of the cumulative density functions. 
Finally, to calculate IRRE, the Insufficient Ramping Resource Probability (IRRP) is calculated using the AFD, as shown in (26). IRRE for each direction (upward or downward flexibility) is the summation of the corresponding IRRP values, as shown in (27).

$$
\begin{gathered}
I R R P_{h,+/-}^{t}=A F D_{h,+/-}\left(D R_{h,+/-}^{t}-1\right) \\
I R R E_{h,+/-}=\sum_{\forall t \in T} I R R P_{h,+/-}^{t}
\end{gathered}
$$

\section{CASE STUdy}

The data of a real isolated power system (of one month) with a peak gross load of $732 \mathrm{MW}$, load factor 0.76 and highest RES penetration in power demand of $90 \%$ are used in this case study to evaluate the impact of a BESS to the operating cost, $\mathrm{CO}_{2}$ emissions, and flexibility.

\section{A. Operating Cost and $\mathrm{CO}_{2}$ Emissions Analysis}

The operating cost and $\mathrm{CO}_{2}$ emissions analysis is performed by solving the UC problem for four different scenarios using the same load, but different BESS capacity, as shown in Table I. Both indices are calculated based on the resulting UC for each scenario and tabulated in Table II.

\begin{tabular}{|c|c|c|c|c|c|c|}
\hline \multirow{2}{*}{ Scenario } & \multirow{2}{*}{$\begin{array}{l}\mathrm{SOC}_{\min } \\
\text { (MWh) }\end{array}$} & \multirow{2}{*}{$\begin{array}{l}\text { SOC }_{\max } \\
\text { (MWh) }\end{array}$} & \multirow{2}{*}{$\begin{array}{c}P_{D i s}^{\max } \\
(\mathbf{M W})\end{array}$} & \multirow{2}{*}{$\begin{array}{l}P_{C h}^{\max } \\
(\mathrm{MW})\end{array}$} & \multicolumn{2}{|c|}{ Efficiency } \\
\hline & & & & & $s_{d}$ & $s_{c}$ \\
\hline 1 & 0 & 0 & 0 & 0 & 0 & 0 \\
\hline 2 & 0 & 40 & 40 & 40 & 0.96 & 0.96 \\
\hline 3 & 0 & 80 & 80 & 80 & 0.96 & 0.96 \\
\hline 4 & 0 & 120 & 120 & 120 & 0.96 & 0.96 \\
\hline
\end{tabular}

TABLE I: BESS SPECIFICATIONS PER SCENARIO

TABLE II: OPERATING COST AND $\mathrm{CO}_{2}$ EMISSIONS

\begin{tabular}{|c|c|c|}
\hline Scenario & Operating Cost $\mathbf{( M € )}$ & $\mathbf{C O}_{2}$ emissions (tons) \\
\hline 1 & 22.466 & 222,786 \\
\hline 2 & 22.445 & 222,568 \\
\hline 3 & 22.433 & 222,367 \\
\hline 4 & 22.425 & 222,270 \\
\hline
\end{tabular}

Based on the results of this study, the operating cost of the power system may be reduced with the integration of a BESS. The cost reduction over the considered period and compared to the first scenario (no BESS), is $0.09 \%, 0.15 \%$, and $0.18 \%$ for Scenario 2, 3, and 4, respectively. Moreover, the $\mathrm{CO}_{2}$ emissions are reduced by $0.09 \%, 0.19 \%$, and $0.23 \%$. The first remark is that the reduction in the operating cost is doubled when the BESS of Scenario 4 is deployed compared to the BESS of Scenario 2, even though the capacity of the BESS is tripled. In other words, a BESS of larger capacity is not always expected to have larger impact on the operating cost. This indicates that an aspect that should be taken into consideration for having maximum benefits from the installation of a BESS is the optimal sizing of a BESS. It should be noted that a more holistic view of the impact of the BESS in terms of cost should take into consideration the initial investment cost, technology, and life expectancy of the BESS. This is currently out of the scope of the paper and it will be investigated in future works.

Regarding the $\mathrm{CO}_{2}$ emissions, it seems that the larger the capacity of the BESS the more the reduction of the $\mathrm{CO}_{2}$ emissions. This is due to the fact that the BESS can be charged both by the power generated by the conventional units as well as the excess RES power that might be available in a given time horizon. In this sense, environmental friendlier power is used for satisfying the generation-demand balance.

\section{B. Flexibility Analysis}

The flexibility analysis for the system is performed using the gross load (for a month) and the BESS capacity of the Scenarios 1 and 4 of Table I. The different penetration levels of RES are presented in Table III, while the main sources involved in this study are the solar, wind, and biomass power. The six different penetration scenarios result in twelve UC solutions when solved for the two BESS capacities $(0$ and $120 \mathrm{MWh})$. The operating cost for each case is shown in Table IV, while the $\mathrm{CO}_{2}$ emissions are depicted in Fig. 1.

TABLE III: RES PENETRATION SCENARIOS

\begin{tabular}{|c|c|c|c|c|}
\hline Scenario & $\begin{array}{c}\text { Ideal } \\
\text { Penetration }\end{array}$ & $\begin{array}{c}\text { Wind } \\
\text { (MW) }\end{array}$ & $\begin{array}{c}\text { Biomass } \\
\text { (MW) }\end{array}$ & $\begin{array}{c}\text { Solar } \\
(\mathbf{M W})\end{array}$ \\
\hline 1 - Reference & $23 \%$ & 157 & 15 & 416 \\
\hline 2 & $25 \%$ & 200 & 23 & 416 \\
\hline 3 & $30 \%$ & 200 & 84 & 416 \\
\hline 4 & $35 \%$ & 200 & 84 & 545 \\
\hline 5 & $40 \%$ & 280 & 84 & 600 \\
\hline 6 & $50 \%$ & 370 & 100 & 750 \\
\hline
\end{tabular}

TABLE IV: OPERATING COST (MILLION €)

\begin{tabular}{|c|c|c|c|c|c|c|}
\hline \multirow{2}{*}{$\begin{array}{c}\text { BESS } \\
\text { Scenario }\end{array}$} & \multicolumn{7}{|c|}{ RES Penetration Scenario } \\
\cline { 2 - 7 } & $\mathbf{1}$ & $\mathbf{2}$ & $\mathbf{3}$ & $\mathbf{4}$ & $\mathbf{5}$ & $\boldsymbol{6}$ \\
\hline 1 & 22.358 & 21.812 & 20.598 & 19.574 & 18.706 & 17.550 \\
\hline 4 & 22.339 & 21.780 & 20.570 & 19.452 & 18.550 & 17.277 \\
\hline
\end{tabular}

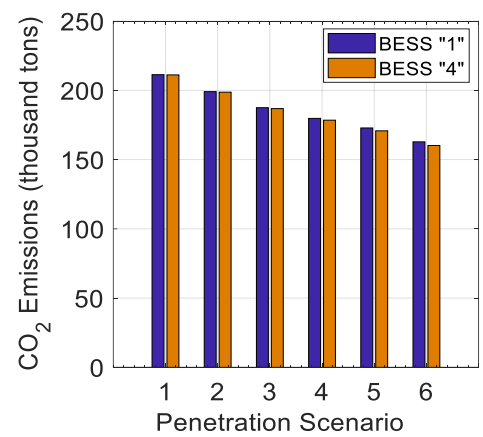

Figure 1. CO2 Emissions under various penetration levels

The results confirm that the BESS impacts positively the power system in terms of cost and emissions, in the case of significantly increased RES penetration. In addition, although the operating cost and the $\mathrm{CO}_{2}$ emissions are reduced when the RES capacity is increased, the reduction is not as high as it is expected to be since security constraints of the system, such as must-run units and spinning reserve, lead to RES curtailments, as indicated in Fig. 2. Based on the SOC of the BESS a great portion of curtailed power can be used for charging the BESS.

For the same scenarios of BESS capacity, the flexibility of the system is evaluated through the calculation of IRRE for each UC solution over a 24-hour time horizon. The downward flexibility does not seem to be an issue for the power system operation since IRRE is zero in all cases. On 
the other hand, the upward flexibility is highly affected by the increased ramping requirements in the absence of storage, as it is depicted in Fig. 3. In particular, the highest value for IRRE is observed in the 5-hour time horizon since the start-up time of most of the considered conventional generating units is 6 hours. Finally, the IRRE is zero when storage is deployed, proving its beneficial effect on the system's flexibility, composing an excellent source of flexibility.

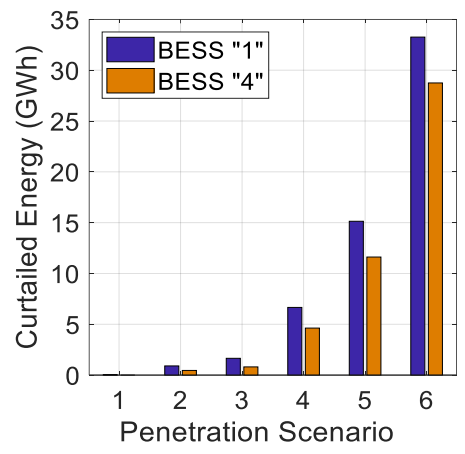

Figure 2. RES curtailment under various penetration levels

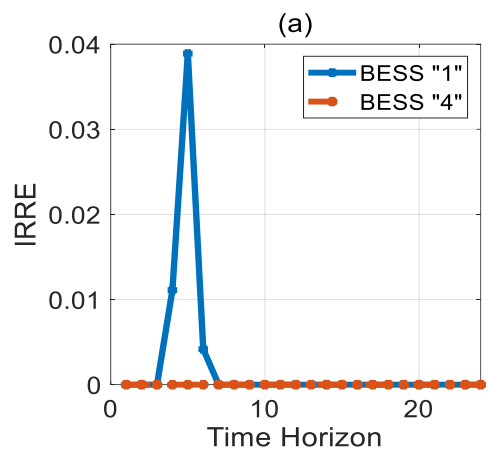

(b)

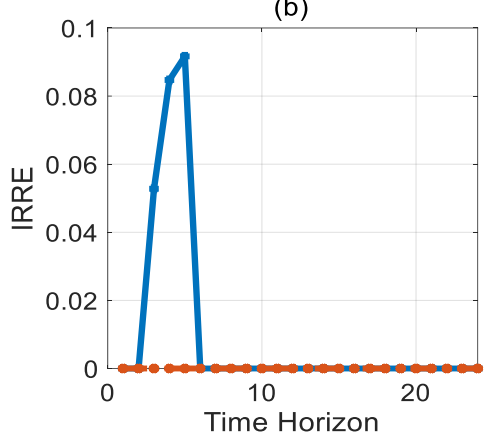

Figure 3: Upward Flexibility (a) Scenario 1 (b) Scenario 6

\section{CONCLUSIONS}

This paper evaluates the economic, environmental and technical impact of a BESS on the operation of an isolated power system with limited flexible resources. For the evaluation of these factors, the UC problem is solved and then the operating cost, $\mathrm{CO} 2$ emissions, and the IRRE are calculated. Different types of BESS are incorporated in a real power system to evaluate their impact on the operating cost and $\mathrm{CO}_{2}$ emissions, indicating that as the size of the BESS increases then the positive impact on both indices is higher; however, in the case of cost, the selection of the optimal BESS size should consider other factors, such as technology type, life expectancy and investment cost. The optimal sizing of the BESS, taking into account these factors, is a study that should be done in the future. Furthermore, in the case of RES penetration increase, the BESS plays a major role in reducing cost and emissions. Lastly, regarding flexibility, it is proved that the upward flexibility is significantly improved when a BESS is deployed based on the IRRE. In this sense, the methodology for calculating the IRRE can be significantly improved, providing more realistic results, by modifying the resources model to include the effect of combined cycle units and more realistic modeling of BESS; this is also going to be investigated in future works. In general, the deployment of BESS in modern power systems is proved as a completely viable solution and could be an effective means to make the power systems greener, more flexible, and more cost-efficient.

\section{REFERENCES}

[1] Z. Yang, H.-C. I. Herbert, F. Tyrone, Y. Fang and E. Kianoush, "Cooperative dispatch of BESS and wind power generation considering carbon emission limitation in Australia," IEEE Transactions on Industrial Informatics, vol. 11, no. 6, pp. 1313-1323, 2015.

[2] J. Yang, L. Zhang, X. Han and M. Wang, "Evaluation of operational flexibility for power system with energy storage," in International Conference on Smart Grid and Clean Energy Technologies (ICSGCE), Chengdu, 2016.

[3] L. Tziovani, M. Savva, M. Asprou, P. Kolios, E. Kyriakides, R. Tapakis, M. Michael and C. Hadjilaou, "Assesing the operational flexibility in power systems with energy storage integration," in Special Session in ISH 2019, No: 1, Flexitranstore, B. Németh, Springer, 2020.

[4] G. N. Psarros, E. G. Karamanou and S. A. Papathanassiou, "Feasibility analysis of centralized storage facilities in isolated grids," IEEE Transactions on Sustainable Energy, vol. 9, no. 4, pp. 1822 - 1832, 2018.

[5] C. Jian, L. Yutian and B. Guannan, " Optimal operating strategy for distribution networks with PV and BESS considering flexible energy storage," in IEEE Power and Energy Society General Meeting (PESGM), Boston, MA, 2016.

[6] N. A. Amoli and A. S. Meliopoulos, " Operational flexibility enhancement in power systems with high penetration of wind power using compressed air energy storage," in Clemson University Power Systems Conference (PSC), Clemson, SC, 2015.

[7] V. Oree and S. S. Hassen, "A composite metric for assessing flexibility available in conventional generators of power systems," Applied Energy, vol. 177, pp. 683-691, 2016.

[8] E. Lannoye, D. Flynn and M. O'Malley, "Evaluation of power system flexibility," IEEE Transactions on Power Systems, vol. 27, no. 2, pp. 922-931, 2012.

[9] D. Jiandong and J. Zhuanting, "Research on improving flexibility of integrated power and gas energy system considering P2G and demand response," in IEEE Conference on Energy Internet and Energy System Integration (EI2), Beijing, 2017.

[10]P. Finn, C. Fitzpatrick, M. Leahy and L. Relihan, "Promotion of wind generated electricity using price responsive demand side management: price prediction analysis for imperfect energy storage," in Proceedings of the 2010 IEEE International Symposium on Sustainable Systems and Technology, Arlington, VA, 2010

[11]Transmission System Operator of Cyprus (TSOC), "RES Penetration," 2018. [Online]. Available: https://www.dsm.org.cy/en//cypruselectrical-system/electrical-energy-generation/energy-generationrecords/yearly/res-penetration. 